\title{
One-Pot Synthesis of Novel Highly Functionalized Furan-Based Polyphenolics
}

Article in Synlett · October 2015

DO : $0.055 / \mathrm{s} 0035560580$

C A ONS

2

8 authors, including:
READS

113

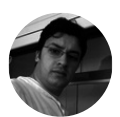

Oualid Talhi

Centre de Recherche Scientifique et Techniq... 31 PUBL CA ONS $72 \mathrm{C}$ A ONS

SEE PROF LE

\section{Gilbert Kirsch}

University of Lorraine

354 PUBL CA ONS 3,210 C A ONS

SEE PROF LE

\section{K. Bachari}

Centre de Recherche Scientifique et Techniq... 125 PUBL CA ONS $460 \mathrm{C}$ A ONS

SEE PROF LE

\section{Artur M S Silva}

University of Aveiro

802 PUBL CA ONS 8,614 C A ONS

SEE PROF LE

Some of the authors of this publication are also working on these related projects:

Project Valorization of Algerian vegetable waste in the wastewater treatment View project 


\section{Complimentary and personal copy}

\section{SYNLETT}

\section{Accounts and Rapid Communications in Chemical Synthesis}

This electronic reprint is provided for noncommercial and personal use only: this reprint may be forwarded to individual colleagues or may be used on the author's homepage. This reprint is not provided for distribution in repositories, including social and scientific networks and platforms.

Publishing House and Copyright:

(C) 2015 by

Georg Thieme Verlag KG

Rüdigerstraße 14

70469 Stuttgart

ISSN 0936-5214 


\section{One-Pot Synthesis of Novel Highly Functionalized Furan-Based Polyphenolics}

\author{
Joana L. C. Sousa ${ }^{a}$ \\ Oualid Talhi*a,c \\ Djenisa H. A. Rocha ${ }^{a}$ \\ Diana C. G. A. Pinto \\ Filipe A. Almeida Paz \\ Khaldoun Bacharic \\ Gilbert Kirsch ${ }^{d}$ \\ Artur M. S. Silva*a \\ a QOPNA, Department of Chemistry, University of Aveiro, \\ 3810-193 Aveiro, Portugal \\ artur.silva@ua.pt \\ oualid.talhi@ua.pt \\ ${ }^{\mathrm{b}} \mathrm{CICECO}$ - Aveiro Institute of Materials, Department of \\ Chemistry, University of Aveiro, 3810-193 Aveiro, Portugal \\ ' Centre de Recherche Scientifique et Technique en Analyses \\ Physico-Chimiques CRAPC, BP384, Bou-Ismail, 42004 \\ Tipaza, Algeria \\ d Laboratoire Structure et Réactivité des Systèmes Moléculaires \\ Complexes, UMR 7565, Université de Lorraine, Avenue du \\ Général Delestraint, 57070 Metz, France
}

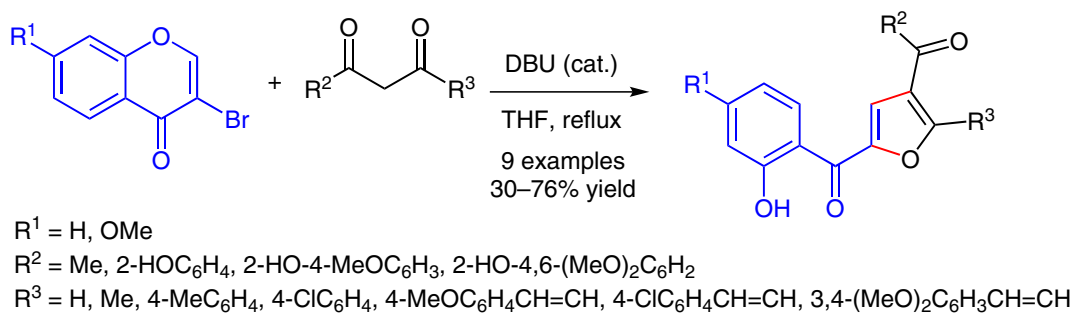

$\mathrm{R}^{2}=\mathrm{Me}, 2-\mathrm{HOC}_{6} \mathrm{H}_{4}, 2-\mathrm{HO}-4-\mathrm{MeOC}_{6} \mathrm{H}_{3}, 2-\mathrm{HO}-4,6-(\mathrm{MeO})_{2} \mathrm{C}_{6} \mathrm{H}_{2}$

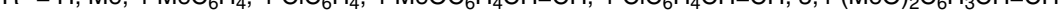

Received: 12.09 .2015

Accepted after revision: 04.10.2015

Published online: 21.10 .2015

DOI: 10.1055/s-0035-1560580; Art ID: st-2015-d0717-I

Abstract Novel, highly functionalized furan-based polyphenolics were prepared. The employed methodology involves a one-pot 1,8-diazabicyclo[5.4.0]undec-7-ene (DBU) catalyzed 1,4-conjugate addition of 1,3-dicarbonyl compounds on 3-bromochromones, furan heterocyclization, and chromanone ring opening.

Key words furan, polyphenols, 1,3-dicarbonyl compounds, 1,4-conjugate addition, ring transformation

The furan heterocycle is found in a variety of synthetic and natural compounds, and some of these present a myriad of biological activities, such as antitumor ${ }^{1}$ and antiviral activity, ${ }^{2}$ among others. ${ }^{3}$ The furan moiety is also considered an important structural unit in polymers. ${ }^{4}$ Furthermore, furfural and 5-hydroxymethylfurfural are basic nonpetroleum chemicals that can be transformed into numerous chemical intermediates of high industrial value. ${ }^{5}$

One of the most important methods for the synthesis of furans is the Paal-Knorr approach, which consists of the acid-catalyzed dehydrative cyclization of 1,4-dicarbonyl compounds. ${ }^{6}$ Domino and multicomponent procedures ${ }^{7}$ and transition-metal-catalyzed synthesis ${ }^{8}$ are other methods that are used for the preparation of furan-based derivatives. Nevertheless, due to the multiple operating reaction steps and chemical-consuming drawbacks associated with the currently reported methods, there remains a need to develop procedures that can be conducted under milder reaction conditions towards furan-based derivatives.

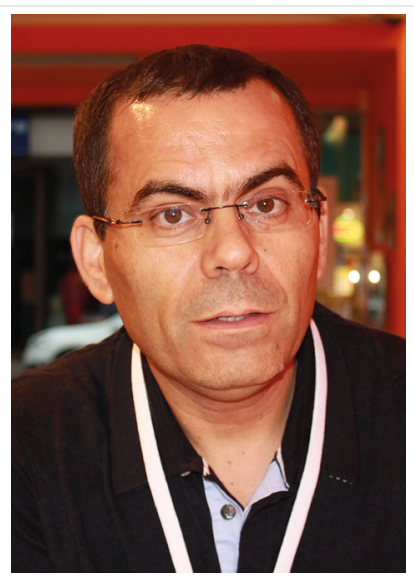

Artur M. S. Silva is a full professor of organic chemistry, University of Aveiro, Portugal. He studied chemistry at the University of Aveiro (Portugal) where he graduated in chemistry physics in 1987. In 1993 he received his PhD in chemistry at Aveiro University, and began his independent career at Aveiro University as an assistant professor in 1994. He was appointed to associate professor with tenure in 1998 and full professor in 2001. He has published over 470 papers and 20 book chapters and has supervised work on $21 \mathrm{PhD}$ and $30 \mathrm{MSc}$ theses. His research interests include the chemistry of polyphenolic and nitrogen heterocyclic compounds, with special emphasis on the development of new synthetic routes, and organocatalytic transformations. Other research passions include the isolation and structural characterization of natural products from diverse terrestrial and marine sources.

Chromones, which are an important family of oxygencontaining heterocyclic compounds, ${ }^{9}$ are desirable and versatile starting materials for the design of a variety of het- 
<smiles>[R]C(=O)CC([R])=O</smiles>

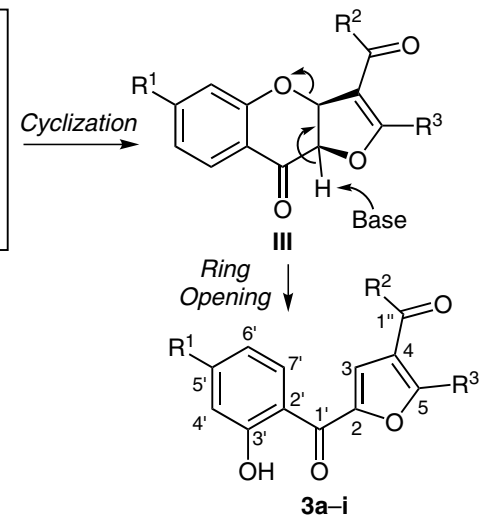

Scheme 1 Synthetic pathway and proposed mechanism for the synthesis of furan-based polyphenolics 3a-i

erocyclic molecules, especially several with important biological applications. ${ }^{10}$ Halogen-containing chromones are a small but interesting group of compounds because of the synthetic transformations they can undergo. ${ }^{11}$ Iaroshenko and Langer recently reported the synthesis of functionalized 2-salicyloylfurans by reacting 3-halochromones with $\beta$-ketoamides using a one-pot protocol. ${ }^{12}$ Moreover, Gammill reported the reaction of 3-bromochromone with dialkyl $\beta$-diketones or $\beta$-ketoesters in the presence of DBN or DBU to prepare trisubstituted furans. ${ }^{13}$
Following our interest in preparing oxygen-containing heterocyclic polyphenolics with potential biological properties using 3-bromochromones as starting materials, ${ }^{14}$ we report herein the synthesis of novel, highly functionalized polysubstituted furan derivatives. The methodology utilized ${ }^{12,13}$ involves the reaction of 3-bromochromones $\mathbf{1 a , b}$ with a series of substituted 1,3-dicarbonyl compounds $\mathbf{2 a -}$ h catalyzed by DBU (Scheme 1,Table 1$).^{15}$

Table 1 Furan-Based Polyphenolics 3a-i and Starting 3-Bromochromones 1a,b and 1,3-Dicarbonyl Compounds 2a-h<smiles>C[13C](C)(C)C</smiles>

$1 \mathrm{a}$

$$
\prod_{2 a}
$$$$
\text { 1,3-Dicarbonyl compound }
$$<smiles>COc1ccc(C(=O)CC(=O)/C=C/c2ccc(OC)c(OC)c2)c(O)c1</smiles>

2b<smiles>[R4]Oc1ccccc1C(=O)CC(=O)/C=C/c1ccc(Cl)cc1</smiles>

\section{Fu}

$3 a$<smiles>CC(=O)c1cc(C(=O)c2ccccc2O)oc1C</smiles><smiles>COc1ccc(C(=O)c2cc(C(=O)c3ccccc3O)oc2/C=C/c2ccc(OC)c(OC)c2)c(O)c1</smiles>

$3 b$<smiles>CC(C)(O)c1ccccc1C(=O)c1cc(C(=O)c2ccccc2O)c(/C=C/c2ccc(Cl)cc2)o1</smiles> 
Table 1 (continued)

3-Bromochromon

1,3-Dicarbonyl compound

Furan product

Yield (\%)<smiles>Cc1ccc(C(=O)CC(=O)c2ccccc2O)cc1</smiles><smiles>Cc1ccc(-c2oc(C(=O)c3ccccc3O)cc2C(=O)c2ccccc2O)cc1</smiles><smiles>COc1ccc(C(=O)CC=O)c(O)c1</smiles>

$2 e$<smiles>COc1ccc(C(=O)c2coc(C(=O)c3ccccc3O)c2)c(O)c1</smiles>

$\mathrm{MeO}$<smiles>CC(=O)CC(C)=O</smiles>

1b<smiles>[Z7]Oc1ccccc1C(=O)CC(C)=O</smiles>

$3 f$<smiles>COc1ccc(C(=O)c2cc(C(C)=O)c(C)o2)c(O)c1</smiles>

$3 g$<smiles>COc1ccc(C(=O)c2cc(C(=O)c3ccccc3O)c(C)o2)c(O)c1</smiles><smiles>COc1ccc(/C=C/c2oc(C(=O)c3ccc(OC)cc3O)cc2C(=O)c2c(O)cc(OC)cc2OC)cc1</smiles>

$2 \mathrm{~h}$<smiles>COc1ccc(/C=C/C(=O)CC(=O)c2c(O)cc(OC)cc2OC)cc1</smiles>

$3 \mathbf{i}$

\footnotetext{
${ }^{a}$ Yield after chromatographic purification and recrystallization.
} 
The best results were achieved when a catalytic amount of base ( 1 drop) was used in THF heated to reflux for 48 hours, with furans 3a-i being obtained in moderate to good yields (30-76\%). Given that, under these conditions, furan derivative 3d was obtained in moderate yield (36\%) and some unreacted 3-bromochromone 1a and 1,3-dicarbonyl compound $\mathbf{2 d}$ remained, we examined the use of longer reaction times and increased quantities of base. However, under these conditions, the formation of the desired furan derivative was not favored; instead, increasing amounts of degradation products were observed. Although unsuccessful, the same study was performed for the derivatives obtained in lower yields.

The results seem to indicate that the reaction is favored by the use of less substituted 1,3-dicarbonyl reagents, which permits access to the corresponding furans $\mathbf{3 a}, \mathbf{3 e}$, and $\mathbf{3 f}$ in good yields (60-76\%). The use of 1,3-dicarbonyl compounds with a styryl group as substituent leads to the corresponding 5-styryl substituted furan derivatives $\mathbf{3 b}$ and 3c ( 48 and $56 \%$, respectively) in better yields than those of 3d and 3h, bearing the 5-phenyl group (36 and 38\%, respectively). The synthetic procedure described herein possesses several advantages, including mild conditions and a onepot protocol, and it is suitable for the construction of a library of important furan-based polyphenolic compounds (Table 1). ${ }^{16}$

As proposed by Gammill, ${ }^{13}$ the reaction presumably follows a tandem process that is initiated by an organobasepromoted 1,4-conjugate addition of $\mathbf{2}$ to $\mathbf{1}$, leading to intermediate I, which is in equilibrium with the corresponding enolic tautomer in the attached 1,3-dicarbonyl moiety II. $^{17}$ This enolic form readily undergoes intramolecular heterocyclization, involving nucleophilic substitution of the bromo leaving group, affording a fused dihydrofuran-chromanone intermediate III. Finally, this intermediate goes through a chromanone ring opening, and is converted into polysubstituted furan $\mathbf{3}$ (Scheme 1).

The most important structural features revealed from analysis of the ${ }^{1} \mathrm{H}$ NMR spectra of furans $\mathbf{3 a} \mathbf{a}-\mathbf{i}$ are the singlet corresponding to the resonance of the $\mathrm{H}-3$ proton at $\delta=$ 7.35-7.61 ppm. However, the furan ring of derivative $\mathbf{3 e}$ displays two doublets at $\delta=7.71(\mathrm{H}-3)$ and $8.20(\mathrm{H}-5) \mathrm{ppm}$ as a result of the small coupling between protons $\mathrm{H}-3$ and $\mathrm{H}-5\left(\mathrm{~J}_{\mathrm{H} 3-\mathrm{H} 5}=0.8 \mathrm{~Hz}\right)$. The $3{ }^{\prime}-\mathrm{OH}$ and $3 "-\mathrm{OH}$ protons of the 2,4-benzoyl substituents appear at high frequency $(\delta=$ $11.88-12.80$ and $11.86-12.03$ ppm, respectively), due to intramolecular hydrogen bonding with the carbonyl groups. Furans $\mathbf{3 b}, \mathbf{3 c}$, and $\mathbf{3 i}$, bearing a 5-styryl group, were mainly characterized by the presence of the $\mathrm{CH}^{\alpha}=\mathrm{CH}^{\beta}$ double bond, which resonates as two doublets at $\delta=7.22-7.37\left(\mathrm{H}^{\alpha}\right)$ and 7.52-7.57 $\left(\mathrm{H}^{\beta}\right)$ ppm, and exhibits a mutual coupling constant $J_{\mathrm{H} \alpha-\mathrm{H} \beta}$ of approximately $16 \mathrm{~Hz}$, typical of the transconfiguration.
The structure of furans 3a-i was further confirmed by their ${ }^{13} \mathrm{C}$ NMR spectra and by analysis of their HSQC and HMBC spectra (Figure 1 shows the main HMBC correlations for 3a). Differentiation between quaternary carbons C-2 $(\delta=148.5-152.0 \mathrm{ppm})$ and $\mathrm{C}-4(\delta=121.1-127.3 \mathrm{ppm})$ was achieved by examining the HMBC correlations that were established with the neighboring protons $\mathrm{H}-3,5-\mathrm{CH}_{3}$ and $\mathrm{H}^{\alpha}$ in the 5-styryl substituted furans (Figure 1). Moreover, in all cases, the $4-\mathrm{C}=\mathrm{O}(\delta=190.1-195.8 \mathrm{ppm})$ was found to be more deshielded than the $2-\mathrm{C}=\mathrm{O}(\delta=182.6-184.8 \mathrm{ppm})$.

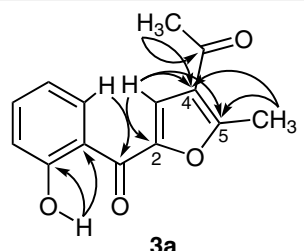

Figure 1 Main HMBC connectivities of furan 3a

The structural features of furans $\mathbf{3 a - i}$ were further investigated by single-crystal X-ray diffraction analysis of compound 3a. This compound produced good-quality single crystals from a (1:1) mixture of hexane and dichloromethane by slow evaporation at $6{ }^{\circ} \mathrm{C}$. Crystallographic studies show that $\mathbf{3 a}$ crystallizes in the centrosymmetric monoclinic $P 2_{1} / n$ space group, ${ }^{18}$ with the molecular unit present in the crystal structure confirming the structure previously indicated by NMR studies (Figure 2).

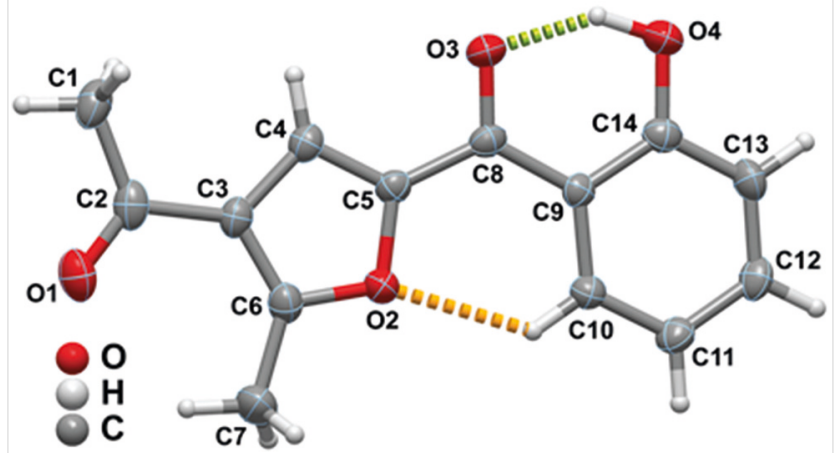

Figure 2 Schematic representation of the molecular unit present in the crystal structure of $3 a$. Non-hydrogen atoms are represented as thermal ellipsoids drawn at the $50 \%$ probability level and hydrogen atoms are shown as small spheres with arbitrary radii. Intramolecular $\mathrm{O}-\mathrm{H} \ldots \mathrm{O}$ and $\mathrm{C}-\mathrm{H} \ldots \mathrm{O}$ hydrogen bonds are depicted as dashed green and orange lines, respectively. Hydrogen-bond geometry: $d_{04 \ldots 03}=2.517(2) \AA$ and $\mathrm{OHO}$ angle $145^{\circ} ; d_{\mathrm{C} 7 \ldots 01}=3.065(3) \AA$ and $\mathrm{CHO}$ angle $126^{\circ}$.

In summary, we have reported the preparation of furanbased polyphenolic derivatives by a one-pot synthetic methodology. This procedure starts with an organobasecatalyzed 1,4-conjugate addition of 1,3-dicarbonyl compounds onto 3-bromochromones and follows a tandem process of furan heterocyclization and chromanone ring open- 
ing. The biological properties of these novel, highly functionalized furan-based polyphenolics will be investigated in due course.

\section{Acknowledgment}

Thanks are due to FCT/MEC for the financial support of the QOPNA research Unit (FCT UID/QUI/00062/2013) and CICECO-Aveiro Institute of Materials (FCT UID/CTM/50011/2013), through national funds and, where applicable, co-financed by the FEDER, within the PT2020 Partnership Agreement, as well as to the Portuguese NMR Network. We would like to thank the General Directorate for Scientific Research and Technological Development-DGRSDT of Algeria for financial support. Further thanks are due to CICECO for funding the purchase of a single-crystal X-ray diffractometer. J.L.C.S. is grateful to FCT for her PhD grant (SFRH/BD/76407/2011).

\section{Supporting Information}

Supporting information for this article is available online at http://dx.doi.org/10.1055/s-0035-1560580.

\section{References and Notes}

(1) (a) Luo, Y.; Wang, K.; Zhang, M.-h.; Zhang, D.-y.; Wu, Y.-c.; Wu, X.-m.; Hua, W.-y. Bioorg. Med. Chem. Lett. 2015, 25, 2421. (b) Diana, P.; Carbone, A.; Barraja, P.; Kelter, G.; Fiebig, H.-H.; Cirrincione, G. Bioorg. Med. Chem. 2010, 18, 4524. (c) Dong, Y.; Shi, Q.; Liu, Y.-N.; Wang, X.; Bastow, K.-H.; Lee, K. F. J. Med. Chem. 2009, 52, 3586.

(2) (a) Yanagita, H.; Urano, E.; Matsumoto, K.; Ichikawa, R.; Takaesu, Y.; Ogata, M.; Murakami, T.; Wu, H.; Chiba, J.; Komano, J.; Hoshino, T. Bioorg. Med. Chem. 2011, 19, 816. (b) Katritzky, A. R.; Tala, S. R.; Lu, H.; Vakulenko, A. V.; Chen, Q.-Y.; Sivapackiam, J.; Pandya, K.; Jiang, S.; Debnath, A. K. J. Med. Chem. 2009, 52, 7631.

(3) (a) Wang, X.-D.; Wei, W.; Wang, P.-F.; Yi, L.-C.; Shi, W.-K.; Xie, Y.-X.; Wu, L.-Z.; Tang, N.; Zhu, L.-S.; Peng, J.; Liu, C.; Li, X.-H.; Tang, S.; Xiao, Z.-P.; Zhu, H.-L. Bioorg. Med. Chem. 2015, 23, 4860. (b) Logoglu, E.; Yilmaz, M.; Katircioglu, H.; Yakut, M.; Mercan, S. Med. Chem. Res. 2010, 19, 490. (c) Mortensen, D. S.; Rodriguez, A. L.; Carlson, K. E.; Sun, J.; Katzenellenbogen, B. S.; Katzenellenbogen, J. A. J. Med. Chem. 2001, 44, 3838.

(4) (a) Montiel-Herrera, M.; Gandini, A.; Goycoolea, F. M.; Jacobsend, N. E.; Lizardi-Mendoza, J.; Recillas-Mota, M.; Argüelles-Monal, W. M. Carbohydr. Polym. 2015, 128, 220. (b) Araya-Hermosilla, R.; Broekhuis, A. A.; Picchioni, F. Eur. Polym. J. 2014, 50, 127. (c) Gandini, A.; Belgagem, M. N. Monomers, Polymers and Composites from Renewable Resources; Elsevier: UK, 2008, 115-152. (d) Gandini, A.; Hariri, S.; Le Nest, J.-F. Polymer 2003, 44, 7565. (e) Gandini, A.; Belgagem, M. N. Prog. Polym. Sci. 1997, 22, 1203.

(5) (a) Tanaka, S.; Ashida, K.; Tatsuta, G.; Mori, A. Synlett 2015, 26, 1496. (b) Chang, F.; Dutta, S.; Becnel, J. J.; Estep, A. S.; Mascal, M. J. Agric. Food Chem. 2014, 62, 476. (c) Sutton, A. D.; Waldie, F. D.; Wu, R.; Schlaf, M.; Silks, L. A. P.; Gordon, J. C. Nat. Chem. 2013, 5, 428. (d) Lange, J.-P.; van der Heide, E.; van Buijtenen, J.; Price, R. ChemSusChem 2012, 5, 150. (e) Corma, A.; Iborra, S.; Velty, A. Chem. Rev. 2007, 107, 2411.
(6) (a) Chen, L.; Du, Y.; Zeng, X.-P.; Shi, T.-D.; Zhou, F.; Zhou, J. Org. Lett. 2015, 17, 1557. (b) Khaghaninejad, S.; Heravi, M. M. In Advances in Heterocyclic Chemistry; Vol. 111; Katritzky, A. R., Ed.; Elsevier: USA, 2014, 95-146. (c) Minetto, G.; Raveglia, L. F.; Sega, A.; Taddei, M. Eur. J. Org. Chem. 2005, 5277. (d) Rao, H. S. P.; Jothilingam, S. J. Org. Chem. 2003, 68, 5392. (e) Stauffer, F.; Neier, R. Org. Lett. 2000, 2, 3535.

(7) (a) Raimondi, W.; Dauzonne, D.; Constantieux, T.; Bonne, D.; Rodriguez, J. Eur. J. Org. Chem. 2012, 6119. (b) Cao, H.; Jiang, H.; Yao, W.; Liu, X. Org. Lett. 2009, 11, 1931. (c) Yadav, J. S.; Reddy, B. V. S.; Shubashree, S.; Sadashiv, K.; Naidu, J. J. Synthesis 2004, 2376.

(8) (a) Cao, H.; Zhan, H.; Cen, J.; Lin, J.; Lin, Y.; Zhu, Q.; Fu, M.; Jiang, H. Org. Lett. 2013, 15, 1080. (b) Liu, W.-B.; Chen, C.; Zhang, Q. Synth. Commun. 2013, 43, 951. (c) Zhou, C.-Y.; Chan, P. W. H.; Che, C.-M. Org. Lett. 2006, 8, 325. (d) Duan, X.-H.; Liu, X.-Y.; Guo, L.-N.; Liao, M.-C.; Liu, W.-M.; Liang, Y.-M. J. Org. Chem. 2005, 70, 6980.

(9) Gaspar, A.; Matos, M. J.; Garrido, J.; Uriarte, E.; Borges, F. Chem. Rev. 2014, 114, 4960

(10) (a) Irgashev, R. A.; Safrygin, A. V.; Ezhikova, M. A.; Kodess, M. I.; Röschenthaler, G.-V.; Sosnovskikh, V. Y. Tetrahedron 2015, 71, 1822. (b) Liu, J.; Li, Z.; Tong, P.; Xie, Z.; Zhang, Y.; Li, Y. J. Org. Chem. 2015, 80, 1632. (c) Esteves, C. I. C.; Santos, C. M. M.; Brito, C. M.; Silva, A. M. S.; Cavaleiro, J. A. S. Synlett 2011, 1403.

(11) Tomé, S. M.; Silva, A. M. S.; Santos, C. M. M. Curr. Org. Synth. 2014, 11, 317.

(12) Savych, I.; Gläsel, T.; Villinger, A.; Sosnovskikh, V. Y.; Iaroshenko, V. O.; Langer, P. Org. Biomol. Chem. 2015, 13, 729.

(13) Gammill, R. B. J. Org. Chem. 1979, 44, 3988.

(14) Santos, C. M. M.; Silva, A. M. S.; Cavaleiro, J. A. S. Eur. J. Org. Chem. 2009, 2642.

(15) Synthesis of Furan-Based Polyphenolics 3a-i; General Procedure: 3-Bromo-4H-chromen-4-ones 1a,b and 1,3-dicarbonyl compounds $\mathbf{2 b}-\mathbf{h}$ were prepared by following previously reported methods. ${ }^{14,19}$ 3-Bromochromone 1a,b $(1.0 \mathrm{mmol})$ and 1,3-dicarbonyl compound $\mathbf{2 a - h}(1.1 \mathrm{mmol})$ were dissolved in THF ( $40 \mathrm{~mL}$ ). DBU (1 drop per $50 \mathrm{mg}$ of starting material) was then added and the resulting reaction mixture was stirred vigorously and heated to reflux. After $48 \mathrm{~h}$, the solvent was evaporated under reduced pressure and the residue was purified by short plug silica gel column chromatography using dichloromethane as eluent. The purified products $\mathbf{3 a}-\mathbf{i}$ were recrystallized from hexane/dichloromethane (1:1) by slow evaporation at $6{ }^{\circ} \mathrm{C}$.

(16) Typical Analytical Data: (4-Acetyl-5-methylfuran-2-yl)(2hydroxyphenyl)methanone (3a): Yield: $171 \mathrm{mg}$ (70\%); yellow crystals; mp 99-100 ${ }^{\circ} \mathrm{C}$ ( Lit. $\left.^{13} \mathrm{mp} 98-100{ }^{\circ} \mathrm{C}\right) .{ }^{1} \mathrm{H}$ NMR $(300.13$ $\left.\mathrm{MHz}, \mathrm{CDCl}_{3}\right): \delta=11.94\left(\mathrm{~s}, 1 \mathrm{H}, 3^{\prime}-\mathrm{OH}\right), 8.19(\mathrm{dd}, J=8.2,1.6 \mathrm{~Hz}$, $\left.1 \mathrm{H}, \mathrm{H}^{-} 7^{\prime}\right), 7.58$ (s, $1 \mathrm{H}, \mathrm{H}-3$ ), 7.54 (ddd, $J=8.5,7.3,1.6 \mathrm{~Hz}, 1 \mathrm{H}$, $\left.\mathrm{H}-5^{\prime}\right), 7.06$ (dd, $\left.J=8.5,1.1 \mathrm{~Hz}, 1 \mathrm{H}, \mathrm{H}-4^{\prime}\right), 6.99$ (ddd, $J=8.2,7.3$, $\left.1.1 \mathrm{~Hz}, 1 \mathrm{H}, \mathrm{H}-6^{\prime}\right), 2.77\left(\mathrm{~s}, 1 \mathrm{H}, 5-\mathrm{CH}_{3}\right), 2.50\left(\mathrm{~s}, 1 \mathrm{H}, 2^{\prime \prime}-\mathrm{CH}_{3}\right)$. ${ }^{13} \mathrm{C}$ NMR (75.47 MHz, $\left.\mathrm{CDCl}_{3}\right): \delta=193.2\left(\mathrm{C}-1^{\prime \prime}\right), 184.4\left(\mathrm{C}-1^{\prime}\right)$, 163.3 (C-3'), 163.2 (C-5), 149.2 (C-2), 136.4 (C-5'), 131.1 (C-7'), 123.4 (C-4), 121.1 (C-3), 119.2 (C-6'), 118.6 (C-4'), 118.5 (C-2'), $29.1\left(2 "-\mathrm{CH}_{3}\right), 15.1\left(5-\mathrm{CH}_{3}\right)$. HRMS $\left(\mathrm{ESI}^{+}\right)$: $\mathrm{m} / \mathrm{z}$ calcd for $\left[\mathrm{C}_{14} \mathrm{H}_{12} \mathrm{O}_{4}+\mathrm{H}\right]^{+}: 245.0808$; found: 245.0821 .

(E)-[4-(2-Hydroxy-4-methoxybenzoyl)-5-(3,4-dimethoxystyryl)furan-2-yl](2-hydroxyphenyl)methanone (3b): Yield: 240 mg (48\%); orange solid; mp $134-135{ }^{\circ} \mathrm{C} .{ }^{1} \mathrm{H}$ NMR $(500.13 \mathrm{MHz}$, $\mathrm{CDCl}_{3}$ ): $\delta=12.59$ (s, $\left.1 \mathrm{H}, 3^{\prime \prime}-\mathrm{OH}\right), 11.95$ (s, $1 \mathrm{H}, 3$ '-OH), 8.22 (dd, $\left.J=8.2,1.6 \mathrm{~Hz}, 1 \mathrm{H}, \mathrm{H}-7^{\prime}\right), 7.64\left(\mathrm{~d}, J=9.0 \mathrm{~Hz}, 1 \mathrm{H}, \mathrm{H}-7^{\prime \prime}\right), 7.59$ (s, $1 \mathrm{H}, \mathrm{H}-3$ ), 7.57 (d, $J=16.5 \mathrm{~Hz}, 1 \mathrm{H}, \mathrm{H}-\beta), 7.56$ (ddd, $J=8.4,7.2$, 
$\left.1.6 \mathrm{~Hz}, 1 \mathrm{H}, \mathrm{H}-5^{\prime}\right), 7.22(\mathrm{~d}, J=16.5 \mathrm{~Hz}, 1 \mathrm{H}, \mathrm{H}-\alpha), 7.16$ (dd, $J=8.4$, $\left.1.9 \mathrm{~Hz}, 1 \mathrm{H}, \mathrm{H}-6^{\prime \prime \prime}\right), 7.10$ (dd, $\left.J=8.4,1.0 \mathrm{~Hz}, 1 \mathrm{H}, \mathrm{H}-4^{\prime}\right), 7.08$ (d, $J=$ $\left.1.9 \mathrm{~Hz}, 1 \mathrm{H}, \mathrm{H}-2^{\prime \prime \prime}\right), 7.02$ (ddd, $\left.J=8.2,7.2,1.0 \mathrm{~Hz}, 1 \mathrm{H}, \mathrm{H}-6^{\prime}\right), 6.89$ (d, $\left.J=8.4 \mathrm{~Hz}, 1 \mathrm{H}, \mathrm{H}-5^{\prime \prime \prime}\right), 6.54$ (d, J = 2.5 Hz, $\left.1 \mathrm{H}, \mathrm{H}-4^{\prime \prime}\right), 6.49$ (dd, $\left.J=9.0,2.5 \mathrm{~Hz}, 1 \mathrm{H}, \mathrm{H}-6^{\prime \prime}\right), 3.95$ (s, $\left.3 \mathrm{H}, 3^{\prime \prime \prime}-\mathrm{OCH}_{3}\right), 3.93$ (s, $3 \mathrm{H}$, 4 "'--OCH $), 3.89$ (s, $\left.3 \mathrm{H}, 5^{\prime \prime}-\mathrm{OCH}_{3}\right) .{ }^{13} \mathrm{C}$ NMR $\left(125.77 \mathrm{MHz}, \mathrm{CDCl}_{3}\right)$ : $\delta=191.9$ (C-1"), 184.3 (C-1'), 166.6 (C-5"), 166.2 (C-3"), 163.3 (C-3'), 159.6 (C-5), 150.8 (C-4"'), 149.3 (C-3"'), 148.9 (C-2), 136.6 (C- $\beta$ ), 136.4 (C-5'), 133.7 (C-7"), 131.0 (C-7'), 128.6 (C1"'), 123.0 (C-3), 122.0 (C-6"'), 121.9 (C-4), 119.3 (C-6'), 118.8 (C-2'), 118.7 (C-4'), 114.0 (C-2"), 112.1 (C- $\alpha), 111.2$ (C-5"'), 109.3 (C-2"'), 108.0 (C-6"), 101.3 (C-4"), 56.02, 56.00 (3"', 4 "'--OCH $\left.{ }_{3}\right), 55.77\left(5^{\prime \prime}-\mathrm{OCH}_{3}\right)$. HRMS $\left(\mathrm{ESI}^{+}\right): \mathrm{m} / \mathrm{z}$ calcd for $\left[\mathrm{C}_{29} \mathrm{H}_{24} \mathrm{O}_{8}+\mathrm{Na}\right]^{+}$: 523.1363; found: 523.1344 .

(E)-[5-(4-Chlorostyryl)furan-2,4-diyl]bis[(2-hydroxyphenyl)methanone] (3c): Yield: $249 \mathrm{mg}(56 \%)$; yellow solid; mp 193$194^{\circ} \mathrm{C} .{ }^{1} \mathrm{H}$ NMR $\left(300.13 \mathrm{MHz}, \mathrm{CDCl}_{3}\right): \delta=11.90\left(\mathrm{~s}, 1 \mathrm{H}, 3^{\prime}-\mathrm{OH}\right)$, 11.86 (s, $\left.1 \mathrm{H}, 3^{\prime \prime}-\mathrm{OH}\right), 8.21$ (dd, $\left.J=8.2,1.5 \mathrm{~Hz}, 1 \mathrm{H}, \mathrm{H}-7^{\prime}\right), 7.72$ (dd, $J=8.1,1.6 \mathrm{~Hz}, 1 \mathrm{H}, \mathrm{H}-7 "), 7.61$ (s, $1 \mathrm{H}, \mathrm{H}-3), 7.60-7.49$ (m, $\left.4 \mathrm{H}, \mathrm{H}-5^{\prime}, 5^{\prime \prime}, \mathrm{H}-3^{\prime \prime \prime}, 5^{\prime \prime \prime}\right), 7.53$ (d, $\left.J=16.3 \mathrm{~Hz}, 1 \mathrm{H}, \mathrm{H}-\beta\right), 7.38$ (d, $J=$ $\left.8.6 \mathrm{~Hz}, 2 \mathrm{H}, \mathrm{H}-2^{\prime \prime \prime}, 6^{\prime \prime \prime}\right), 7.37$ (d, $\left.J=16.3 \mathrm{~Hz}, 1 \mathrm{H}, \mathrm{H}-\alpha\right), 7.12-7.09$ (m, $\left.2 \mathrm{H}, \mathrm{H}-4^{\prime}, 4^{\prime \prime}\right), 7.03$ (ddd, $\left.J=8.1,7.2,1.2 \mathrm{~Hz}, 1 \mathrm{H}, \mathrm{H}-6^{\prime \prime}\right), 6.96$ (ddd, $\left.J=8.2,7.3,1.1 \mathrm{~Hz}, 1 \mathrm{H}, \mathrm{H}-6^{\prime}\right) .{ }^{13} \mathrm{C}$ NMR $\left(75.47 \mathrm{MHz}, \mathrm{CDCl}_{3}\right.$ ): $\delta=193.5\left(\mathrm{C}-1^{\prime \prime}\right), 184.4\left(\mathrm{C}-1^{\prime}\right), 163.4\left(\mathrm{C}-3^{\prime}\right), 163.0\left(\mathrm{C}-3^{\prime \prime}\right), 159.2$ (C-5), 149.3 (C-2), 137.0 (C-5"), 136.6 (C-5'), 135.6 (C-1"'), 135.5

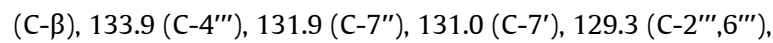
128.8 (C-3"',5"'), 122.7 (C-3), 122.5 (C-4), 119.9 (C-2"), 119.4 (C$\left.6^{\prime}\right), 119.3\left(\mathrm{C}-6^{\prime \prime}\right), 118.8\left(\mathrm{C}-4^{\prime}, 4^{\prime \prime}\right), 118.7\left(\mathrm{C}-2^{\prime}\right), 114.5(\mathrm{C}-\alpha)$. HRMS $\left(\mathrm{ESI}^{+}\right): \mathrm{m} / \mathrm{z}$ calcd for $\left[\mathrm{C}_{26} \mathrm{H}_{17} \mathrm{O}_{5} \mathrm{Cl}+\mathrm{H}\right]^{+}:$445.0837; found: 445.0833.

(17) Liu, W.; Jiang, H.; Zhang, M.; Qi, C. J. Org. Chem. 2010, 75, 966.

(18) Crystal Data for 3a: $\mathrm{C}_{14} \mathrm{H}_{12} \mathrm{O}_{4} ; M=244.24$; monoclinic; space group $P 2_{1} / n ; \quad Z=4 ; \quad a=7.3330(9) \AA, \quad b=10.5928(14) \AA$, $c=15.0866(19) \AA, \quad \beta=90.962(4)^{\circ} ; \quad V=1171.7(3) \AA^{3} ; \quad \mu($ Mo$\left.\mathrm{K}_{\alpha}\right)=0.102 \mathrm{~mm}^{-1} ; D_{\mathrm{c}}=1.385 \mathrm{~g} \mathrm{~cm}^{-3}$; colorless needle; crystal size $0.28 \times 0.10 \times 0.08 \mathrm{~mm}^{3}$. Of a total of 8785 reflections collected, 2126 were independent $\left(R_{\mathrm{int}}=0.0685\right)$. Final $R 1=0.0494$ $[I>2 \sigma(I)]$ and $w R 2=0.1286$ (all data). Data completeness to $\theta=25.24^{\circ}, 99.2 \%$. Crystallographic data for $\mathbf{3 a}$ has been deposited into the Cambridge Crystallographic Data Centre with the deposition number CCDC 1409624.

(19) (a) Davies, S. G.; Mobbs, B. E.; Goodwin, C. J.J. Chem. Soc., Perkin Trans. 1 1987, 2597. (b) Pinto, D. C. G. A.; Silva, A. M. S.; Almeida, L. M. P. M.; Cavaleiro, J. A. S.; Lévai, A.; Patonay, T. J. Heterocyclic Chem. 1998, 35, 217. (c) Rocha, D. H. A. Estudos de síntese e transformação de cromonas e 4-quinolonas. Ph.D. Thesis, University of Aveiro, 2015, pp. 124 and 125. (d) Santos, C. M. M. Transformações de cetonas $\alpha, \beta$ - e $\alpha, \beta, \gamma, \delta$-insaturadas. Novas rotas de síntese de 2,3-diarilxantonas. Ph.D. Thesis, University of Aveiro, 2007, pp. 368 and 385. 